\title{
Search for hep solar neutrinos and the diffuse supernova neutrino background using all three phases of the Sudbury Neutrino Observatory
}

B. Aharmim, ${ }^{8}$ S. N. Ahmed, ${ }^{16}$ A. E. Anthony, ${ }^{18, b}$ N. Barros, ${ }^{15,10}$ E. W. Beier, ${ }^{15}$ A. Bellerive,${ }^{5}$ B. Beltran, ${ }^{1}$ M. Bergevin,,${ }^{9,7}$ S. D. Biller, ${ }^{14}$ E. Blucher, ${ }^{6}$ R. Bonventre, ${ }^{2,9}$ K. Boudjemline, ${ }^{5,16}$ M. G. Boulay, ${ }^{16, d}$ B. Cai, ${ }^{16}$ E. J. Callaghan, ${ }^{2,9}$ J. Caravaca, ${ }^{2,9}$ Y. D. Chan, ${ }^{9}$ D. Chauhan, ${ }^{8, e}$ M. Chen, ${ }^{16}$ B. T. Cleveland, ${ }^{14}$ G. A. Cox, ${ }^{20}$ X. Dai, ${ }^{16,14,5}$ H. Deng, ${ }^{15, f}$ F. B. Descamps, ${ }^{2,9}$ J. A. Detwiler, ${ }^{9, g}$ P. J. Doe, ${ }^{20}$ G. Doucas, ${ }^{14}$ P.-L. Drouin, ${ }^{5}$ M. Dunford, ${ }^{15, h}$ S. R. Elliott, ${ }^{11,20}$ H. C. Evans, ${ }^{16, a}$ G. T. Ewan, ${ }^{16}$ J. Farine,${ }^{8,5}$ H. Fergani ${ }^{14}{ }^{14}$ F. Fleurot, ${ }^{8}$ R. J. Ford,${ }^{17,16}$ J. A. Formaggio, ${ }^{13,20}$ N. Gagnon,${ }^{20,11,9,14}$ K. Gilje, ${ }^{1}$ J. TM. Goon, ${ }^{12}$ K. Graham, ${ }^{5,16}$ E. Guillian, ${ }^{16}$ S. Habib, ${ }^{1}$ R. L. Hahn, ${ }^{4}$ A. L. Hallin, ${ }^{1}$ E. D. Hallman, ${ }^{8}$ P. J. Harvey, ${ }^{16}$ R. Hazama, ${ }^{20, i}$ W. J. Heintzelman, ${ }^{15}$ J. Heise, ${ }^{3,11,16, j}$ R. L. Helmer,${ }^{19}$ A. Hime,${ }^{11}$ C. Howard, ${ }^{1}$ M. Huang, ${ }^{18,8}$ P. Jagam, ${ }^{7}$ B. Jamieson, ${ }^{3, k}$ N. A. Jelley, ${ }^{14}$ M. Jerkins, ${ }^{18}$ K. J. Keeter,,${ }^{17,1}$ J. R. Klein, ${ }^{18,15}$ L. L. Kormos, ${ }^{16, m}$ M. Kos, ${ }^{16, n}$ C. Kraus, ${ }^{16,8}$ C. B. Krauss, ${ }^{1}$ A. Krüger, ${ }^{8}$ T. Kutter, ${ }^{12}$ C. C. M. Kyba, ${ }^{15,0}$ K. Labe, ${ }^{6, p}$ B. J. Land, ${ }^{2,9}$ R. Lange, ${ }^{4}$ A. LaTorre, ${ }^{6}$ J. Law, ${ }^{7}$ I. T. Lawson, ${ }^{17,7}$ K. T. Lesko, ${ }^{9}$ J. R. Leslie, ${ }^{16}$ I. Levine, ${ }^{5,9}$ J. C. Loach, ${ }^{14,9}$ R. MacLellan, ${ }^{16, r}$ S. Majerus ${ }^{14}$ H. B. Mak ${ }^{16}$ J. Maneira, ${ }^{10}$ R. D. Martin,,${ }^{16,9}$ A. Mastbaum $\odot,{ }^{15,6, \mathrm{~s}}$ N. McCauley, ${ }^{15,14, t}$ A. B. McDonald, ${ }^{16}{ }^{\text {S. R. McGee }},{ }^{20}$ M. L. Miller, ${ }^{13, g}$ B. Monreal, ${ }^{13, \mathrm{u}}$ J. Monroe, ${ }^{13, v}$ B. G. Nickel, ${ }^{7}$ A. J. Noble, ${ }^{16,5}$ H. M. O’Keeffe, ${ }^{14, \mathrm{~m}}$ N. S. Oblath, ${ }^{20,13, \mathrm{w}}$ C. E. Okada, ${ }^{9, \mathrm{x}}$ R. W. Ollerhead, ${ }^{7}$ G. D. Orebi Gann, ${ }^{2,15,9}$ S. M. Oser, ${ }^{3,19}$ R. A. Ott, ${ }^{13, y}$ S. J. M. Peeters, ${ }^{1, z}$ A. W. P. Poon, ${ }^{9}$ G. Prior, ${ }^{10,9}$ S. D. Reitzner, ${ }^{7, a a}$ K. Rielage, ${ }^{11,20}$ B. C. Robertson, ${ }^{16}$ R. G. H. Robertson, ${ }^{20}$ M. H. Schwendener, ${ }^{8}$ J. A. Secrest, ${ }^{15, b b}$ S. R. Seibert, ${ }^{18,11,15, \mathrm{cc}}$ O. Simard, ${ }^{5, d d}$ D. Sinclair, ${ }^{5,19}$ P. Skensved, ${ }^{16}$ T. J. Sonley, ${ }^{13, \mathrm{e}}$ L. C. Stonehill, ${ }^{11,20}$ G. Tešić, ${ }^{5}$ N. Tolich, ${ }^{20}$ T. Tsui, ${ }^{3, e e}$ R. Van Berg,${ }^{15}$ B. A. VanDevender ${ }^{20, w}$ C. J. Virtue,${ }^{8}$ B. L. Wall, ${ }^{20}$ D. Waller, ${ }^{5}$

H. Wan Chan Tseung, ${ }^{14,20}$ D. L. Wark, ${ }^{14, f f}$ J. Wendland, ${ }^{3}$ N. West, ${ }^{14}{ }_{5}$. F. Wilkerson, ${ }^{20, g g}$ J. R. Wilson,,${ }^{14, \text { hh }}$ T. Winchester, ${ }^{20}$ A. Wright, ${ }^{16}$ M. Yeh, ${ }^{4}$ F. Zhang, ${ }^{5, i i}$ and K. Zuber ${ }^{14, j j}$

(SNO Collaboration)

\author{
${ }^{1}$ Department of Physics, University of Alberta, Edmonton, Alberta, T6G 2R3, Canada \\ ${ }^{2}$ Physics Department, University of California at Berkeley, Berkeley, California 94720-7300, USA \\ ${ }^{3}$ Department of Physics and Astronomy, University of British Columbia, Vancouver, BC V6T 1Z1, Canada \\ ${ }^{4}$ Chemistry Department, Brookhaven National Laboratory, Upton, New York 11973-5000, USA \\ ${ }^{5}$ Ottawa-Carleton Institute for Physics, Department of Physics, Carleton University, \\ Ottawa, Ontario K1S 5B6, Canada \\ ${ }^{6}$ Department of Physics, University of Chicago, Chicago, Illinois 60637, USA \\ ${ }^{7}$ Physics Department, University of Guelph, Guelph, Ontario N1G 2W1, Canada \\ ${ }^{8}$ Department of Physics and Astronomy, Laurentian University, Sudbury, Ontario P3E 2C6, Canada \\ ${ }^{9}$ Institute for Nuclear and Particle Astrophysics and Nuclear Science Division, Lawrence Berkeley \\ National Laboratory, Berkeley, California 94720-8153, USA \\ ${ }^{10}$ Laboratório de Instrumentação e Física Experimental de Partículas, \\ Avenida Elias Garcia 14, 1²,1000-149 Lisboa, Portugal \\ ${ }^{11}$ Los Alamos National Laboratory, Los Alamos, New Mexico 87545, USA \\ ${ }^{12}$ Department of Physics and Astronomy, Louisiana State University, Baton Rouge, Louisiana 70803, USA \\ ${ }^{13}$ Laboratory for Nuclear Science, Massachusetts Institute of Technology, \\ Cambridge, Massachusetts 02139, USA \\ ${ }^{14}$ Department of Physics, University of Oxford, Denys Wilkinson Building, Keble Road, \\ Oxford OX1 3RH, United Kingdom \\ ${ }^{15}$ Department of Physics and Astronomy, University of Pennsylvania, \\ Philadelphia, Pennsylvania 19104-6396, USA \\ ${ }^{16}$ Department of Physics, Queen's University, Kingston, Ontario K7L 3N6, Canada \\ ${ }^{17}$ SNOLAB, Lively, ON P3Y 1N2, Canada \\ ${ }^{18}$ Department of Physics, University of Texas at Austin, Austin, Texas 78712-0264, USA \\ ${ }^{19}$ TRIUMF, 4004 Wesbrook Mall, Vancouver, BC V6T 2A3, Canada \\ ${ }^{20}$ Center for Experimental Nuclear Physics and Astrophysics, and Department of Physics, \\ University of Washington, Seattle, Washington 98195, USA
}

\footnotetext{
${ }^{\mathrm{a}}$ Deceased.

${ }^{\mathrm{b}}$ Present address: Global Development Lab, U.S. Agency for International Development, Washington, D.C.

${ }^{c}$ Present address: Lawrence Livermore National Laboratory, Livermore, CA.
} 
(Received 15 July 2020; accepted 1 September 2020; published 29 September 2020)

A search has been performed for neutrinos from two sources, the hep reaction in the solar $p p$ fusion chain and the $\nu_{e}$ component of the diffuse supernova neutrino background (DSNB), using the full dataset of the Sudbury Neutrino Observatory with a total exposure of 2.47 kton-years after fiducialization. The hep search is performed using both a single-bin counting analysis and a likelihood fit. We find a best-fit flux that is compatible with solar model predictions while remaining consistent with zero flux, and set a onesided upper limit of $\Phi_{\text {hep }}<30 \times 10^{3} \mathrm{~cm}^{-2} \mathrm{~s}^{-1}$ [90\% credible interval (CI)]. No events are observed in the DSNB search region, and we set an improved upper bound on the $\nu_{e}$ component of the DSNB flux of $\Phi_{\nu_{e}}^{\mathrm{DSNB}}<19 \mathrm{~cm}^{-2} \mathrm{~s}^{-1}(90 \% \mathrm{CI})$ in the energy range $22.9<E_{\nu}<36.9 \mathrm{MeV}$.

DOI: 10.1103/PhysRevD.102.062006

\section{INTRODUCTION}

Solar neutrinos produced in the $p p$ fusion cycle have been studied extensively by several experiments [1-6]. However, the highest energy branch in this cycle, the hep reaction $\left[{ }^{3} \mathrm{He}\left(p, e^{+} \nu_{e}\right)^{4} \mathrm{He}\right]$, has yet to be directly detected. With a predicted branching ratio of $\sim 2 \times 10^{-7}$, the flux expected on Earth in the BSB05(GS98) solar model is (7.93 \pm 1.23$) \times 10^{3} \mathrm{~cm}^{-2} \mathrm{~s}^{-1}$ $[7,8]$. As the hep reaction has the highest end point energy of all solar neutrinos, and occurs at a relatively large radius in the Sun, an observation may provide sensitivity to nonstandard solar models in addition to completing our picture of the $p p$ chain neutrino fluxes.

\footnotetext{
${ }^{\mathrm{d}}$ Present address: Department of Physics, Carleton University, Ottawa, Ontario, Canada.

${ }^{\mathrm{e}}$ Present address: SNOLAB, Lively, ON, Canada.

${ }^{\mathrm{f}}$ Present address: Rock Creek Group, Washington, D.C.

${ }^{g}$ Present address: Center for Experimental Nuclear Physics and Astrophysics, and Department of Physics, University of Washington, Seattle, WA.

${ }^{\mathrm{h}}$ Present address: Ruprecht-Karls-Universität Heidelberg, Im Neuenheimer Feld 227, Heidelberg, Germany.

${ }^{i}$ Present address: Research Center for Nuclear Physics, Osaka, Japan.

${ }^{\mathrm{j}}$ Present address: Sanford Underground Research Laboratory, Lead, SD.

${ }^{\mathrm{k}}$ Present address: Department of Physics, University of Winnipeg, Winnipeg, Manitoba, Canada.

${ }^{\mathrm{l}}$ Present address: Black Hills State University, Spearfish, SD.

${ }^{\mathrm{m}}$ Present address: Physics Department, Lancaster University, Lancaster, UK.

${ }^{\mathrm{n}}$ Present address: Pelmorex Corp., Oakville, ON.

${ }^{\circ}$ Present address: GFZ German Research Centre for Geosciences, Potsdam, Germany.

${ }^{\mathrm{p}}$ Present address: Department of Physics, Cornell University, Ithaca, NY.

${ }^{\mathrm{q}}$ Present address: Department of Physics and Astronomy, Indiana University, South Bend, IN.

${ }^{\mathrm{r}}$ Present address: Department of Physics and Astronomy, University of Kentucky, Lexington KY.

${ }^{s}$ Present address: Department of Physics and Astronomy, Rutgers University, Piscataway, NJ.

tPresent address: Department of Physics, University of Liverpool, Liverpool, UK.

"Present address: Department of Physics, Case Western Reserve University, Cleveland, OH.

${ }^{\mathrm{v}}$ Present address: Department of Physics, Royal Holloway University of London, Egham, Surrey, UK.

${ }^{w}$ Present address: Pacific Northwest National Laboratory, Richland, WA.

${ }^{\mathrm{x}}$ Present address: Nevada National Security Site, Las Vegas, NV.

${ }^{\mathrm{y}}$ Present address: Department of Physics, University of California, Davis, CA.

${ }^{\mathrm{z}}$ Present address: Department of Physics and Astronomy, University of Sussex, Brighton, UK.

${ }^{\text {aa }}$ Present address: Fermilab, Batavia, IL.

${ }^{\mathrm{bb}}$ Present address: Department of Physics, Georgia Southern University, Statesboro, GA.

${ }^{c c}$ Present address: Continuum Analytics, Austin, TX.

${ }^{\mathrm{dd}}$ Present address: National Bank of Canada, Montreal, QC, Canada.

${ }^{e e}$ Present address: Kwantlen Polytechnic University, Surrey, BC, Canada.

${ }^{\mathrm{ff}}$ Additional address: Rutherford Appleton Laboratory, Chilton, Didcot, UK.

${ }^{\text {gg }}$ Present address: Department of Physics, University of North Carolina, Chapel Hill, NC.

${ }^{\mathrm{hh}}$ Present address: Department of Physics, King's College London, London, UK.

${ }^{i i}$ Present address: Laufer Center, Stony Brook University, Stony Brook, NY.

${ }^{\mathrm{j}}$ Present address: Institut für Kern- und Teilchenphysik, Technische Universität Dresden, Dresden, Germany.
}

Published by the American Physical Society under the terms of the Creative Commons Attribution 4.0 International license. Further distribution of this work must maintain attribution to the author(s) and the published article's title, journal citation, and DOI. Funded by $S C O A P^{3}$. 
Also expected in the energy range above the end point of the ${ }^{8} \mathrm{~B}$ solar neutrino spectrum is the diffuse supernova neutrino background (DSNB), the isotropic neutrino flux from past core-collapse supernovae $[9,10]$. A measurement of the DSNB would provide new data on supernova dynamics averaged over these past core-collapse events, which would constrain models and provide context for nearby core collapse supernova events detectable on an individual basis, such as SN1987A [11-14]. In particular, the total flux provides a measure of the average supernova luminosity in neutrinos, and the spectrum is dependent on the temperature at the surface of last scattering. The DSNB signal remains undetected, and the Sudbury Neutrino Observatory (SNO) experiment provides unique sensitivity to the $\nu_{e}$ component of the flux [15].

A previous search for the hep and DSNB neutrinos with the SNO detector used data from the first operating phase, 306.4 live days with a heavy water $\left(\mathrm{D}_{2} \mathrm{O}\right)$ target [16]. The present work extends that counting analysis to the full SNO dataset across all operating phases, and additionally a spectral fit is performed. Section II briefly introduces the SNO detector. Next, Sec. III describes the dataset, event selection, and the counting and fit-based analysis methods. Finally, results are presented in Sec. IV.

\section{THE SNO DETECTOR}

The SNO detector [17] consisted of a target volume enclosed within a transparent acrylic sphere $6 \mathrm{~m}$ in radius, viewed by 9456 inward-looking 8-inch photomultiplier tubes (PMTs) at a radius of $8.4 \mathrm{~m}$, as illustrated in Fig. 1. The acrylic vessel and the structure supporting the PMTs (PSUP) were suspended in a water-filled cavity, which was additionally instrumented with outward-looking PMTs to provide an active veto system. In order to shield from cosmic ray muons and from the neutrons and radioisotopes resulting from muon interactions, the detector was located deep underground with a $5890 \pm 94$ meter water equivalent rock overburden at the Inco (now Vale) Creighton mine near Sudbury, Ontario, Canada.

The detector operated in three distinct phases, differing in the primary mechanism for neutron detection. In the first phase, the detector was loaded with a very low background heavy water $\left(\mathrm{D}_{2} \mathrm{O}\right)$ target. With the $\mathrm{D}_{2} \mathrm{O}$ target, $\mathrm{SNO}$ was sensitive to charged current (CC), neutral current (NC), and elastic scattering (ES) channels:

$$
\begin{aligned}
\nu_{e}+d & \rightarrow p+p+e^{-}-1.44 \mathrm{MeV}(\mathrm{CC}), \\
\nu+d & \rightarrow p+n+\nu-2.22 \mathrm{MeV}(\mathrm{NC}), \\
\nu+e^{-} & \rightarrow \nu+e^{-}(\mathrm{ES}) .
\end{aligned}
$$

The hep and DSNB searches benefit in particular from the enhancement by a factor of about 100 of the CC cross section with respect to that for ES, and from the fact that in

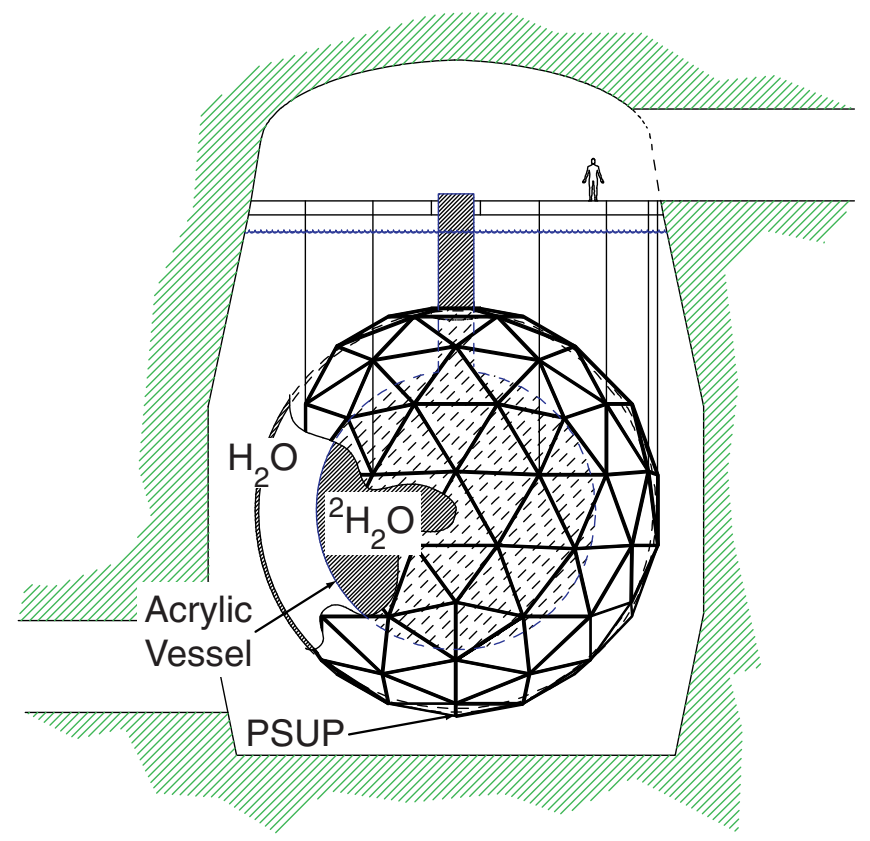

FIG. 1. The SNO detector [5].

the CC interaction, the outgoing electron energy is strongly correlated with the incoming neutrino energy.

In SNO's second operational phase, the $\mathrm{D}_{2} \mathrm{O}$ was doped with $0.2 \% \mathrm{NaCl}$ by mass, to take advantage of the improved neutron capture cross section on $\mathrm{Cl}$ and the higher energy and more isotropic deexcitation $\gamma$ cascade. In the third phase, the $\mathrm{NaCl}$ was removed and an array of ${ }^{3} \mathrm{He}$ proportional counters (NCDs) was deployed to further improve neutron detection. In all three phases, backgrounds due to atmospheric neutrino interactions are reduced significantly via coincidence tagging of final state neutrons.

\section{ANALYSIS}

We performed a single-bin counting analysis in two different energy ranges, for the hep and DSNB neutrino signals. Additionally, a maximum likelihood fit was used to extend the sensitivity of the hep search. The following sections describe the dataset, event selection criteria, and systematic uncertainties common to the counting and likelihood analyses, and then introduce those techniques.

\section{A. Data selection}

This analysis makes use of the entire SNO dataset, across all three operational phases, with data collected between November 1999 and November 2006. Table I indicates the live time for each phase, corresponding to a total exposure of 2.47 kilotonne-years after fiducialization. We adopted a pseudo-blind approach in which the analysis was tuned on Monte Carlo simulations, then validated on one third of the data randomly sampled in short blocks of time uniformly distributed throughout the phases. Finally, with cuts and 
TABLE I. Duration and live time for each operational phase.

\begin{tabular}{lccc}
\hline \hline Phase & Target & Dates & Live time \\
\hline I & $\mathrm{D}_{2} \mathrm{O}$ & $11 / 1999-5 / 2001$ & $306.4 \mathrm{~d}$ \\
II & $\mathrm{D}_{2} \mathrm{O}+0.2 \% \mathrm{NaCl}$ & $7 / 2001-8 / 2003$ & $478.6 \mathrm{~d}$ \\
III & $\mathrm{D}_{2} \mathrm{O}+\mathrm{NCDs}$ & $11 / 2004-11 / 2006$ & $387.2 \mathrm{~d}$ \\
\hline \hline
\end{tabular}

parameters having been fixed, the full dataset was reopened for this analysis.

The set of signal candidate events follows from three stages of event selection. First, entire runs (approximately 8 hour blocks of live time) are accepted or rejected based on detector conditions. The same selection is applied as in Ref. [18] for phase I and Ref. [5] for phase III. For phase II, the selection from Ref. [19] is extended to include periods with higher than average levels of $\mathrm{Rn}$ or activated $\mathrm{Na}$, which presented important backgrounds for the low energy threshold ${ }^{8} \mathrm{~B}$ oscillation analyses but are insignificant for the higher-threshold hep and DSNB searches.

Next, a set of low-level cuts are applied, which address instrumental background events as well as coincidences with bursts of events or tagged muons. The instrumental backgrounds are caused by detector effects, for example high-voltage discharge of a PMT, or electronic pickup. Such events tend to have distinct signatures, such as correlations in the physical locations of electronics channels, which are very different from signal events. For each phase, the same set of low-level cuts is used as in previous work [5], as these have been extensively validated and tuned to optimize signal efficiency. For this analysis, signal-like events are further required to be isolated in time: any candidate event occurring within $250 \mathrm{~ms}$ of another candidate event is rejected. This includes coincidences with any event with a reconstructed vertex within a $6 \mathrm{~m}$ fiducial volume and a kinetic energy above $4 \mathrm{MeV}$, a trigger of the external veto, or (in phase III only) a detected signal in the NCD array. This reduces background classes that produce coincident electrons, neutrons, or photons, and in particular targets Michel electrons following low-energy muons or nuclear deexcitation photons and atmospheric neutrino $\mathrm{CC}$ electrons with neutron followers.

Finally, a series of high level criteria have been developed based on reconstructed observables, which discriminate the signals of interest from other physics backgrounds. The signature of a signal hep or DSNB neutrino interaction is a single electronlike Cherenkov ring originating within $550 \mathrm{~cm}$ of the detector center. This fiducial volume is chosen to reduce backgrounds associated with $\gamma$ rays and other backgrounds due to the materials surrounding the target volume. Signal Cherenkov rings are highly anisotropic, at a level quantified by the variable $\beta_{14}$ previously described in Ref. [20]. The fraction of PMTs hit within a narrow prompt time window is calculated as the in-time ratio (ITR). This variable can discriminate between wellreconstructed single-ring events or multiring events due to a pileup of interactions or particles. To further discriminate single electronlike events, three Kolmogorov-Smirnov (KS) tests are used. The first simply tests the compatibility of the azimuthal distribution of hits around the reconstructed direction relative to a flat distribution. The second test is a two-dimensional extension that includes the polar angle and compares to a probability distribution derived from calibration data, accounting for energy dependence in the polar angle and solid angle effects in the azimuthal angle. A final test compares the time-of-flight corrected PMT hit times for hits inside the Cherenkov ring to a template distribution also extracted from calibration data. Cuts on these parameters have been adjusted relative to previous SNO analyses as described in Sec. III D, as both the energy regime $(>15 \mathrm{MeV})$ and the objectives (rejection of atmospheric neutrino backgrounds) differ. The distributions in these high-level observables are validated by comparing simulations to data in the low-energy sideband below the hep region of interest and to calibration data using a signal-like ${ }^{8} \mathrm{Li}$ source [21].

\section{B. Monte Carlo simulation}

The detailed microphysical detector model used in previous SNO measurements, SNOMAN [5,17], was again employed for this analysis. SNOMAN was used to generate solar neutrino events, propagate final state particles through the detector geometry, and simulate the optical, triggering, and electronics response of the detector. The SNOMAN Monte Carlo contains run-by-run detector state information, tracking changes over time. All Monte Carlo was produced with at least 500 times the statistics expected in data.

For atmospheric neutrinos above $100 \mathrm{MeV}$, we use GENIE v2.12.2 [22,23] using the default model set, and the Bartol04 flux predictions [24], interpolated between the solar minimum and maximum according to the dates of each operational phase. The final state particles from GENIE are then input into SNOMAN for propagation through the full detector simulation. Atmospheric neutrino oscillations are applied using best-fit parameters in a model which samples an ensemble of baselines from the neutrino production height distributions of Gaisser and Stanev [25].

A model for final-state $\gamma$ resulting from interactions with oxygen is included in the GENIE simulation [23,26,27]. However, this does not include a potential background due to a $15.1-\mathrm{MeV} \gamma$ produced in deexcitation of ${ }^{12} \mathrm{C}^{*}$ following neutrino interactions on ${ }^{16} \mathrm{O}$. Here, we take a sample of such untagged $\gamma$ events following neutral current quasielastic (NCQE) interactions from a NUANCE (version 3r009) simulation, which uses the calculation of Ejiri [26], and scale according to the relative NCQE cross section in GENIE.

To model low-energy $\left(E_{\nu}<100 \mathrm{MeV}\right)$ atmospheric neutrino interactions, we use the flux given by Battistoni et al. [28]; fluxes for the SNO location have been provided 
by the authors. For this subdominant background, which represents $\sim 2 \%(\sim 4 \%)$ of the overall atmospheric neutrino background in the hep (DSNB) energy region of interest, only $\nu_{e}$ and $\bar{\nu}_{e}$ are simulated, and the fluxes at the solar minimum (when the background is largest) are used. This simulation is performed directly in SNOMAN. We note that the low- and high-energy atmospheric neutrino fluxes are the same as those used in the 2006 SNO hep and DSNB search analysis [16].

\section{Signals and backgrounds}

For the hep solar neutrino signal, we use the spectrum computed by Bahcall and Ulrich $[29,30]$ and use the BSB05 (GS98) flux of 7.93(1 \pm 0.155$) \times 10^{3} \mathrm{~cm}^{-2} \mathrm{~s}^{-1}[7,8]$ as a benchmark. The primary background for the hep search is due to electrons from ${ }^{8} \mathrm{~B}$ solar neutrino interactions, at a level that depends on the shape of the spectrum near the end point. The spectral shape from Winter et al. [31] is used, and oscillations are applied according to a three-neutrino oscillation model using best-fit parameters [32]. The ${ }^{8} \mathrm{~B}$ solar neutrino flux is based on a three-phase analysis of SNO ${ }^{8} \mathrm{~B}$ solar neutrino data, identical to that presented in Ref. [5] except that an upper energy threshold at $10 \mathrm{MeV}$ was applied to eliminate any contamination from a possible hep signal. The extracted ${ }^{8} \mathrm{~B}$ flux is $\Phi_{8_{\mathrm{B}}}=(5.26 \pm$ $0.16(\text { stat })_{-0.13}^{+0.11}$ (syst) $) \times 10^{6} \mathrm{~cm}^{-2} \mathrm{~s}^{-1}$, consistent with the published value.

The DSNB signal is modeled as an isotropic $\nu_{e}$ source using a benchmark energy spectrum and total flux. We use the model of Beacom and Strigari [15] with $T=6 \mathrm{MeV}$, which predicts a total flux of $\Phi_{\nu_{e}}^{\mathrm{DSNB}}=0.66 \mathrm{~cm}^{-2} \mathrm{~s}^{-1}$ in the energy range $22.9<E_{\nu}<36.9 \mathrm{MeV}$.

Backgrounds due to isotropic light emission from the acrylic vessel [19] have also been studied using a dedicated event selection and Monte Carlo. The background contamination depends on the choice of fiducial volume, and is constrained to the negligible level of $<0.01$ events within our energy regions of interest for the chosen cut of $550 \mathrm{~cm}$. Atmospheric neutrinos and associated ${ }^{12} \mathrm{C}^{*}$ backgrounds are modeled as described in III B. According to the GENIE simulation, the dominant source of atmospheric background is from decay at rest of muons below or near the Cherenkov threshold. These are predominantly produced directly in $\nu_{\mu}$ and $\bar{\nu}_{\mu}$ CC interactions, with a small contribution from decays of subthreshold CC- and NCproduced $\pi^{ \pm} \rightarrow \mu^{ \pm} \rightarrow e^{ \pm}$. Decays of subthreshold muons account for the majority of the background for the DSNB search, while the atmospheric backgrounds for the hep search are subdominant and result from a mix of subthreshold muon decays, $15.1-\mathrm{MeV} \gamma$ rays, and other $\mathrm{NC}$ interactions. The direct production of untagged low-energy electrons in $\nu_{e} \mathrm{CC}$ interactions accounts for a small portion of the background, $\lesssim 10 \%$ in each case.

\section{Counting analysis}

Within each energy region of interest (ROI) for the single-bin counting analysis, 1D cuts on high level features are simultaneously tuned to optimize the search sensitivity in Monte Carlo, with further adjustments to minimize the impact of the systematic uncertainties on the shapes of the observable distributions. The hep energy ROI of $14.3<$ $T_{\text {eff }}<20 \mathrm{MeV}$ and DSNB ROI of $20<T_{\text {eff }}<40 \mathrm{MeV}$ are chosen to optimize signal-to-background ratio while maximizing signal acceptance, following the procedure described in Ref. [16]. The signal efficiency of the highlevel cuts is validated using calibration datasets as shown in Fig. 2. Within the hep ROI, the high level and burst cuts together reduce the atmospheric neutrino backgrounds by $97 \%$, with a signal efficiency of $\sim 99 \%$.

For the purposes of this cut-based analysis, confidence intervals are constructed using a Bayesian framework in which we construct intervals from a Poisson likelihood function marginalized over the expected background distribution. This function is defined as

$$
\begin{aligned}
- & \log \mathcal{L}\left(\mu, b \mid n, \hat{b}, \sigma_{b}\right) \\
= & \mu+b+\log \Gamma(n+1)-n \times \log (\mu+b) \\
& +\frac{1}{2} \frac{(b-\hat{b})^{2}}{\sigma_{b}^{2}},
\end{aligned}
$$

where $\mu$ is the true signal mean, $b$ the true background rate, $n$ the observed number of events, $\hat{b}$ the mean background

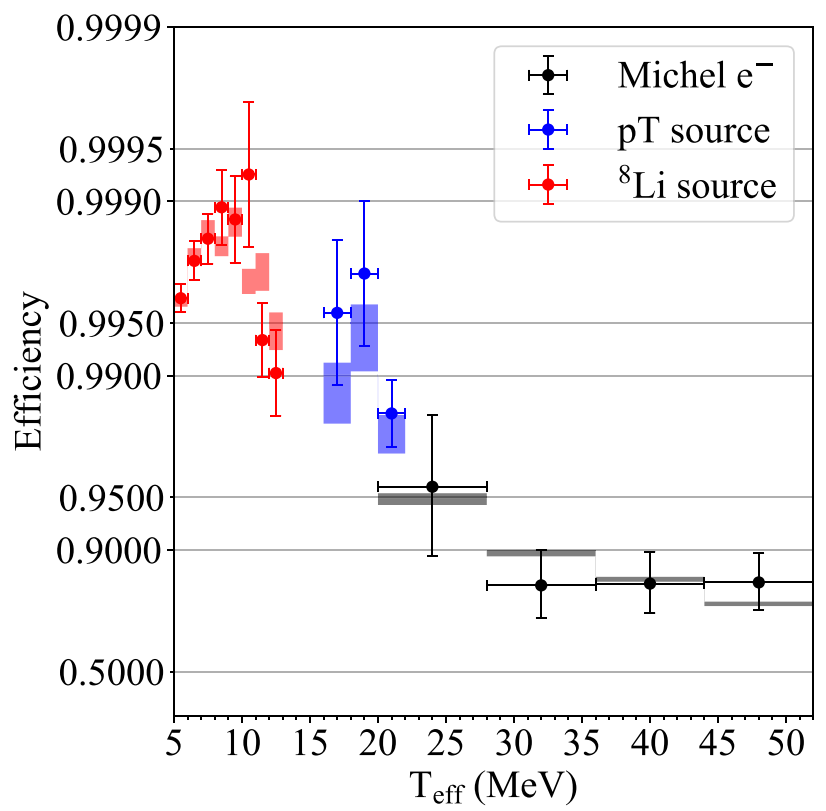

FIG. 2. Efficiency of the high-level event selection cuts for phase I, compared between calibration sample data (points) and Monte Carlo (shaded boxes). The calibration samples include deployed ${ }^{8} \mathrm{Li}$ [21] and $p T$ [33] sources and Michel electrons from muons that stop and decay inside the detector. 
expectation, and $\sigma_{b}$ the Gaussian uncertainty on $b$. In constructing this likelihood function, we have chosen a step function prior that is constant for $\mu>0$. Integrating over the background parameter $b$ yields $-\log \mathcal{L}(\mu \mid n)$, which is treated as a posterior probability distribution function (PDF) for $\mu$ and used to construct intervals. For a confidence level $\alpha$, a two-sided interval $\mathcal{C}$ is defined by the highest posterior density region (HPDR), i.e., adding points $\mu$ in order of their posterior probability density until $\sum_{\mathcal{C}} \mathcal{L}(\mu \mid n) \geq \alpha$. One-sided intervals are constructed by direct integration of $\mathcal{L}$ to determine the smallest $\mu^{\prime}$ such that $\sum_{0}^{\mu^{\prime}} \mathcal{L}(\mu \mid n) \geq \alpha$.

\section{E. Likelihood analysis}

In order to leverage the energy dependence of the signal spectra and lower the threshold for the hep search, an unbinned maximum likelihood fit was also performed. The fit considers all three phases simultaneously, with the ${ }^{8} \mathrm{~B}$ and hep fluxes held constant across time, as well as the overall atmospheric neutrino flux normalization after accounting for differences across the solar minimum and maximum. The dominant systematic uncertainties are varied in the fit using Gaussian pull terms, and include the oscillation parameters $\theta_{12}$ and $\Delta m_{12}^{2}$ as well as the energy scale and resolution model parameters and angular and $\beta_{14}$ resolutions, which are treated as uncorrelated. The fit uses three-dimensional PDFs, binned in reconstructed energy ( $T_{\text {eff }}$, ten bins, $10-20 \mathrm{MeV}$ ), the angle relative to the Sun $\left(\cos \theta_{\text {sun }}\right.$, ten bins, $\left.-1-1\right)$, and the isotropy parameter $\left(\beta_{14}, 15\right.$ bins, $\left.-0.12-0.95\right)$. PDFs are constructed for ${ }^{8} \mathrm{~B}$ CC electrons, ${ }^{8} \mathrm{~B}$ ES electrons, hep CC electrons, hep ES electrons, and atmospheric neutrino interactions for each phase. The relative normalizations of the CC and ES components for each signal are fixed. The cuts described previously are applied to data and Monte Carlo prior to PDF construction and fitting; these include the fiducial volume, ITR, three KS probability figures of merit, and low-level cuts. In contrast to the counting analysis, energy and isotropy are observables in the fit.

The full negative log likelihood function optimized in the fit is of the form [34]

$$
-\log \mathcal{L}(\mathbf{r}, \Delta)=\sum_{j=1}^{M} \tilde{N}_{j}(\mathbf{r}, \Delta)-\sum_{i=1}^{N} \log \left(\sum_{j=1}^{M} \tilde{N}_{j}(\mathbf{r}, \Delta) \times \mathbf{P}_{j}\left(\mathbf{x}_{i}, \Delta\right)\right)+\frac{1}{2} \sum_{k=1}^{M^{\prime}} \frac{\left(r_{k}-\bar{r}_{k}\right)^{2}}{\sigma_{r_{k}}^{2}}+\frac{1}{2} \sum_{m=1}^{s} \frac{\left(\Delta_{m}-\bar{\Delta}_{m}\right)^{2}}{\sigma_{\Delta_{m}}^{2}},
$$

where the first term corresponds to the total normalization constraint, the second to the unbinned likelihood given the PDFs, and the final two terms represent Gaussian uncertainties on rate and systematic parameters. In Eq. (2), $\mathbf{P}_{j}$ are PDFs for each signal, which are binned in the set of observables $\mathbf{x}$. These PDFs are constructed from Monte Carlo events that have been modified according to a set of $s$ systematic parameters $\Delta$, with associated Gaussian uncertainties $\sigma_{\Delta}$.

The parameters $\mathbf{r}$ correspond to signal rates, which may be correlated across event types, e.g., in the case of the hep flux which scales both the CC and ES hep event rates. Thus, the signal rates are related to the expected number of events of a particular type $(\tilde{N})$ by an efficiency matrix $\epsilon$ defined such that $\tilde{N}_{i}=\epsilon_{i}^{j} r_{j}$. The Gaussian uncertainties associated with signal rates are denoted $\sigma_{r} . M^{\prime}$ is simply the number of rate parameters which are externally constrained.

The efficiency matrix $\epsilon$ also accounts for events shifting into or out of the boundary of the analysis window (a volume $V$ in observable space) following the application of a systematic transformation $S$. This is handled by the inclusion of a weighting factor $\left|\left\{\mathbf{x}_{i} \mid S\left(\mathbf{x}_{i}, \Delta\right) \in V\right\}\right|$.

The fit was performed using a Markov chain Monte Carlo (MCMC), and a number of metrics were used to evaluate fit quality and convergence. These included a check of statistical compatibility of parameter distributions within subdivisions of the MCMC random walk, and a toy Monte Carlo to evaluate the goodness of fit through a $\chi^{2}$ hypothesis test. Additional validation included signal injection tests varying the hep flux from 0.01-10 times model predictions.

\section{F. Systematic uncertainties}

A number of systematic effects are important within these analyses. The primary background to the hep search is electrons from ${ }^{8} \mathrm{~B}$ solar neutrino interactions, where the spectrum is affected by the energy response modeling as well as the flux normalization and intrinsic shape. The flux uncertainty is taken from the three-phase fit to low-energy SNO data described in Sec. III C, and the shape is varied within the uncertainties provided by Winter et al. [31]. For solar neutrinos, the uncertainties in the oscillation parameters and the $\nu-d \mathrm{CC}$ cross section are also included. To address the energy response modeling, which affects all signals and backgrounds, uncertainties are derived from fits to deployed calibration sources and samples of Michel electrons; this procedure is described in Sec. IIIF 1. Uncertainties impacting atmospheric neutrino backgrounds are detailed in Sec. III F 2. The major systematic uncertainties impacting the analyses are summarized in Table II.

\section{Detector response}

In order to calibrate the response in the detector across an energy range up to $40 \mathrm{MeV}$, several event samples were 
TABLE II. Systematic uncertainties. Values apply to all three phases except as noted for those in the lower part of the table.

\begin{tabular}{|c|c|c|c|}
\hline \multicolumn{2}{|l|}{ Parameter } & \multicolumn{2}{|r|}{ Magnitude } \\
\hline \multicolumn{2}{|l|}{ Vertex accuracy } & \multicolumn{2}{|r|}{$2.9 \%[5]$} \\
\hline \multicolumn{2}{|l|}{ Vertex resolution } & \multicolumn{2}{|r|}{$2.4 \mathrm{~cm} \mathrm{[5]}$} \\
\hline \multicolumn{2}{|l|}{ Angular resolution } & \multicolumn{2}{|r|}{$2 \%$} \\
\hline \multicolumn{2}{|l|}{${ }^{8} \mathrm{~B}$ flux } & \multicolumn{2}{|r|}{ See Sec. III C } \\
\hline \multicolumn{2}{|l|}{${ }^{8} \mathrm{~B} \nu_{e}$ spectrum } & \multicolumn{2}{|r|}{ Ref. [31] } \\
\hline$\nu$ Mixing parameters & & \multicolumn{2}{|r|}{ Ref. [32] } \\
\hline \multicolumn{4}{|l|}{ Atmospheric $\nu$ flux } \\
\hline$E_{\nu}>100 \mathrm{MeV}$ & & \multirow{2}{*}{\multicolumn{2}{|c|}{$\begin{array}{l}10 \%[24] \\
25 \%[28]\end{array}$}} \\
\hline$E_{\nu}<100 \mathrm{MeV}$ & & & \\
\hline \multicolumn{4}{|l|}{ Cross sections } \\
\hline $\mathrm{CC} \nu-D$ & & \multirow{3}{*}{\multicolumn{2}{|c|}{$\begin{array}{c}1.2 \% \\
\text { See Sec. III F } 2 \\
100 \%\end{array}$}} \\
\hline Atmospheric $\nu$ & & & \\
\hline \multirow{2}{*}{$15.1 \mathrm{MeV} \gamma$ rays } & & & \\
\hline & Phase I & Phase II & Phase III \\
\hline Live time & $0.006 \%$ & $0.021 \%$ & $0.36 \%$ \\
\hline \multicolumn{4}{|l|}{ Energy scale } \\
\hline$T_{\mathrm{eff}}=14.3 \mathrm{MeV}$ & $0.61 \%$ & $0.55 \%$ & $0.82 \%$ \\
\hline$T_{\text {eff }}=20.0 \mathrm{MeV}$ & $0.71 \%$ & $0.65 \%$ & $0.86 \%$ \\
\hline Energy resolution scale & $1.60 \%$ & $1.71 \%$ & $1.37 \%$ \\
\hline
\end{tabular}

compared against SNOMAN Monte Carlo predictions. The vertex reconstruction is described in Refs. [35] (phases I and II) and [36] (phase III), and based on this we include a $2.4 \mathrm{~cm}$ uncertainty on reconstructed position resolution, and an overall $2.9 \%$ fiducial volume uncertainty. Additionally, a $2 \%$ uncertainty on the angular resolution for ES events is modeled as a scaling via a parameter $\Delta_{\theta}[5]$ :

$$
(\cos \theta)^{\prime}=1+(\cos \theta-1)\left(1+\Delta_{\theta}\right)
$$

where $(\cos \theta)^{\prime}$ outside the interval $[-1,1]$ are assigned a random value within that interval.

In each of the three phases, a large sample of $6.13-\mathrm{MeV} \gamma$ rays from a deployed ${ }^{16} \mathrm{~N}$ source provided the primary calibration. Additionally, a $p T$ source in phase I provided a sample of $19.8-\mathrm{MeV} \gamma$ rays [33]. To extend the model to higher energies, samples of Michel electrons from decays of stopping cosmic ray muons were selected and fit to a response model allowing an energy-dependent fractional energy scaling $\left(\Delta_{S}^{(i)}\right)$ and shift in resolution $(\Delta R)$ :

$$
\begin{aligned}
T_{\text {eff }}^{\prime}= & T_{\text {eff }}+\left(\Delta_{S}^{(0)}+\Delta_{S}^{(1)} \cdot T_{\text {eff }}\right) \cdot T_{\text {eff }} \\
& +\Delta_{R} \cdot\left(T_{\text {eff }}-T_{\text {true }}\right) .
\end{aligned}
$$

The parameters were extracted using a maximum likelihood fit to the Michel electron samples for each phase, subject to prior constraints based on the deployed source measurements. The extracted parameters are given in Table IV. We find that the parameters are consistent with zero, confirming that the initial ${ }^{16} \mathrm{~N}$-based energy calibration provides a reasonable estimate of energy across the regions of interest, and the correlated errors in each phase indicate the magnitude of systematic shifts that remain compatible with the higher-energy calibration samples. This provides a data-driven constraint on the smearing of the spectrum of electrons produced by ${ }^{8} \mathrm{~B}$ solar neutrino interactions, which forms a dominant background for the hep search.

Additionally, a similar model including a linear scaling and resolution was applied to the shape of the isotropy parameter $\beta_{14}$, with $\Delta_{S}^{(0)}=\Delta_{R}=4.2 \times 10^{-3}$ for all three phases, based on measurements with the ${ }^{16} \mathrm{~N}$ calibration source [5]. Finally, the contribution of any non-Gaussian (flat) tails in the energy response was constrained to the level of $\lesssim 10^{-3}$ events in the energy region of interest based on samples of events from the deployed ${ }^{8} \mathrm{Li}$ source [21], which has a $\beta$ spectrum similar to that of the ${ }^{8} \mathrm{~B}$ solar neutrinos.

\section{Atmospheric neutrinos}

Two main classes of uncertainty affect the atmospheric neutrinos: the flux uncertainty, which is taken to be $25 \%$ [28] and 10\% [24] for low $(<0.1 \mathrm{GeV})$ and high (0.1$10 \mathrm{GeV}$ ) energies, respectively, and the cross sections. The cross section uncertainties are evaluated through event reweighting, by simultaneously varying the parameters in the default GENIE model set (see Ref. [23]) within their respective uncertainties to produce an ensemble of weights corresponding to different model hypotheses.

To validate the modeling of atmospheric neutrino interactions, a sample of fully-contained atmospheric neutrino events was selected. These events are required to have 200-5000 PMTs hit, no activity in the veto region, and must not follow an event tagged with a $\mu$ entering the detector. These requirements provide a high-purity sample of contained atmospheric neutrino candidate events with $T_{\text {eff }} \geq 25 \mathrm{MeV}$ that is independent from the signal selection. Starting from this selection, we search for timecoincident follower events, which mainly consist of Michel electrons $(\Delta t<20 \mu \mathrm{s})$ and neutrons $(\Delta t>20 \mu \mathrm{s})$. These follower events must pass all analysis cuts and have an energy $5<T_{\text {eff }}<100 \mathrm{MeV}$. For the selected events, we compare the multiplicity and timing of coincidences as well as the energy, position, isotropy, and other high-level observables between the atmospheric Monte Carlo and data, and find good agreement within the flux and cross section modeling uncertainties of the GENIE Monte Carlo simulation. Table III provides the total number of atmospheric neutrino candidate follower events, compared to the Monte Carlo expectation.

Additionally, a search was performed for events in the energy range $35<T_{\text {eff }}<70 \mathrm{MeV}$, where Michel electrons from atmospheric neutrinos are expected. With all 
TABLE III. Data/Monte Carlo comparisons for number of followers in selected atmospheric neutrino event candidate events. Followers with $\Delta t<(>) 20 \mu$ s are primarily Michel electrons (neutrons).

\begin{tabular}{|c|c|c|c|c|c|c|}
\hline & \multicolumn{2}{|c|}{ Phase I } & \multicolumn{2}{|c|}{ Phase II } & \multicolumn{2}{|c|}{ Phase III } \\
\hline & Data & $\mathrm{MC}$ & Data & $\mathrm{MC}$ & Data & $\mathrm{MC}$ \\
\hline All followers & 59 & $59.19 \pm 11.52$ & 184 & $180.77 \pm 26.70$ & 72 & $62.04 \pm 10.30$ \\
\hline$\Delta t<20 \mu \mathrm{s}$ & 25 & $30.42 \pm 7.06$ & 31 & $48.09 \pm 9.23$ & 39 & $39.25 \pm 7.49$ \\
\hline$\Delta t>20 \mu \mathrm{s}$ & 34 & $28.77 \pm 6.89$ & 153 & $132.68 \pm 20.90$ & 33 & $22.80 \pm 5.56$ \\
\hline
\end{tabular}

event selection cuts applied, six isolated events were observed, with a Monte Carlo expectation of 3.7 ( $p$-value $17 \%)$. The event rates are consistent across phases, with one event observed ( 0.8 expected) in phase I, three observed (1.3 expected) in phase II, and two observed (1.6 expected) in phase III. Relaxing the time coincidence cuts, we find one, two, and three additional events in phases I, II, and III, respectively. Of these six events, one is followed by a neutron candidate event. The other five are preceded within a few $\mu$ s by a low-energy event, of which three are consistent with deexcitation photons from the primary neutrino interaction, and two are most likely to be near-threshold atmospheric neutrino-induced muons. Extending to higher energies, $70<T_{\text {eff }}<100 \mathrm{MeV}$, we find one additional electronlike event in phase II, which appears in isolation. Further details of these events may be found in Table VI in the Appendix.

\section{RESULTS}

\section{A. Counting analysis}

Within the sensitivity-optimized energy regions of interest for the hep and DSNB signals, we performed a single-bin counting analysis as introduced in Sec. III D. The energy spectra for selected events are shown in Fig. 3. The total signal and background expectations in the 14.3-20 MeV hep energy ROI are $3.09 \pm 0.12$ and $13.89 \pm 1.09$, respectively, with 22 events observed. Nearly all the background in the hep $\mathrm{ROI}$ is due to ${ }^{8} \mathrm{~B}$ solar neutrinos. In the DSNB ROI, 0.08 signal events and 2.58 background events are expected, with zero events observed. The distribution across phases is given in Table V.

TABLE IV. Energy response model parameters extracted from maximum likelihood fits to calibration sample data in each phase.

\begin{tabular}{lrrr}
\hline \hline Parameter & \multicolumn{1}{c}{ Phase I } & \multicolumn{1}{c}{ Phase II } & \multicolumn{1}{c}{ Phase III } \\
\hline Normalization & $135 \pm 12.2$ & $213 \pm 14.8$ & $172 \pm 13.0$ \\
$\Delta_{S}^{(0)} / 10^{-3}$ & $-5.20 \pm 7.21$ & $-0.01 \pm 6.14$ & $1.25 \pm 10.2$ \\
$\Delta_{S}^{(1)} / 10^{-3}$ & $0.44 \pm 0.42$ & $-0.16 \pm 0.37$ & $-0.16 \pm 0.43$ \\
$\Delta_{R}^{(0)} / 10^{-2}$ & $1.83 \pm 1.60$ & $2.38 \pm 1.71$ & $1.61 \pm 1.37$ \\
\hline \hline
\end{tabular}

The uncertainties on the total three-phase signal and background expectations are correlated $\left(r_{h e p}=0.83\right.$, $r_{\text {DSNB }}=0.12$ ), and are obtained using an ensemble of 500 three-phase pseudo-experiments with systematic parameters randomly sampled according to their correlated uncertainties. The dominant source of uncertainty in the hep region is the energy response modeling, due to the steeply falling tail of the ${ }^{8} \mathrm{~B}$ solar neutrino backgrounds. This model is constrained using data spanning the energy range as described in Sec. III F 1.

The majority of candidate events, 13 of 22 , occurred during phase III. These events appear signal-like in all respects, and consistency with background is observed in sidebands with respect to energy and all other high-level observables. According to toy Monte Carlo studies, the probability of observing a statistical fluctuation of at least this magnitude in any one phase is approximately 8\%.

Applying the Bayesian procedure described in Sec. III D yields an $68.3 \%$ credible interval $(\mathrm{CI})$ of $\Phi_{\text {hep }}=(9.6-$ $33) \times 10^{3} \mathrm{~cm}^{-2} \mathrm{~s}^{-1}$; however, as the probability of a statistical fluctuation of this magnitude is significant, we set a one-sided upper limit of

$$
\Phi_{\text {hep }}<40 \times 10^{3} \mathrm{~cm}^{-2} \mathrm{~s}^{-1}(90 \% \mathrm{CI}) .
$$

For comparison, in the previous phase I analysis two events were observed with $0.99 \pm 0.09$ signal and $3.13 \pm$ 0.60 background events expected; this resulted in a $90 \% \mathrm{CL}$ frequentist upper limit on the hep flux of $23 \times 10^{3} \mathrm{~cm}^{-2} \mathrm{~s}^{-1}$ [16].

Of the 2.58 events expected in the DSNB ROI, 2.47 are due to high-energy $\left(E_{\nu}>100 \mathrm{MeV}\right)$ atmospheric neutrinos. $82 \%$ of these are CC interactions, of which $80 \%$ are due to the decay of muons below the Cherenkov threshold, and in $10 \%$ an isolated electron is directly produced in a $\nu_{e}$ CC interaction. Of the $18 \% \mathrm{NC}$ contribution, $\sim 75 \%$ are due to subthreshold muon decays following charged meson production. The remaining 0.11 expected events are due to low-energy $\left(E_{\nu}<100 \mathrm{MeV}\right)$ atmospheric neutrinos, with about $90 \% \nu_{e}$ and $10 \% \bar{\nu}_{e}$. The median experiment in a Monte Carlo ensemble provides $90 \%$ CI sensitivity to signals at least 52 times larger than the benchmark Beacom and Strigari $T=6 \mathrm{MeV}$ model. With an apparent downward fluctuation, zero events are observed, and we set an 

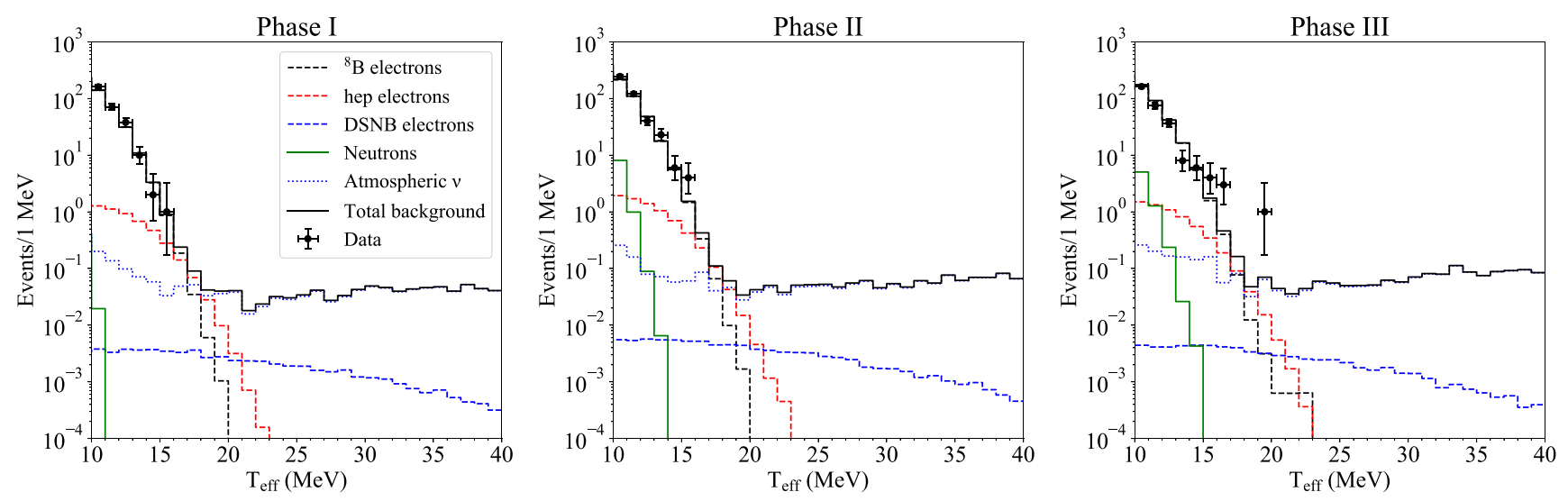

FIG. 3. Reconstructed energy spectra for each phase.

upper limit of 29 times the model prediction, corresponding to DSNB $\nu_{e}$ flux of $\Phi_{\nu_{e}}^{\mathrm{DSNB}}<19 \mathrm{~cm}^{-2} \mathrm{~s}^{-1}(90 \% \mathrm{CI}$ ) in the energy range $22.9<E_{\nu}<36.9 \mathrm{MeV}$. The dominant source of systematic uncertainty in the DSNB ROI is the $10 \%$ normalization uncertainty for the flux of atmospheric neutrinos with $E_{\nu}>100 \mathrm{MeV}$.

\section{B. Likelihood analysis}

For the hep search, we additionally performed a likelihood fit as described in Sec. III E. One-dimensional projections of the best fit in the observable dimensions $T_{\text {eff }}, \beta_{14}$, and $\cos \theta_{\text {sun }}$ are shown in Fig. 5. We note that the shape of the $\cos \theta_{\text {sun }}$ is determined by the $\nu_{e}$ ES and CC cross sections; in the former the outgoing electron direction is strongly correlated with the incoming neutrino direction, while in the latter it is moderately anticorrelated. The quality of the fit was evaluated using a $\chi^{2}$ test based on an effective test statistic distribution derived using a toy Monte Carlo, yielding a $p$ value of $16.0 \%$ considering statistical errors only.

Bayesian credible intervals are obtained as within the counting analysis, by marginalizing over all other parameters. The $1 \sigma$ and $90 \%$ credible intervals are shown in

TABLE V. Summary of expected and observed events for each ROI and phase in the counting analysis.

\begin{tabular}{lccc}
\hline \hline & $\begin{array}{c}\text { Expected } \\
\text { signal }\end{array}$ & $\begin{array}{c}\text { Expected } \\
\text { background }\end{array}$ & $\begin{array}{c}\text { Events } \\
\text { observed }\end{array}$ \\
\hline Phase I hep & $0.84 \pm 0.08$ & $3.14 \pm 0.63$ & 3 \\
Phase II hep & $1.28 \pm 0.06$ & $5.37 \pm 0.65$ & 6 \\
Phase III hep & $0.98 \pm 0.05$ & $5.38 \pm 0.52$ & 13 \\
Total hep & $3.09 \pm 0.12$ & $13.89 \pm 1.09$ & 22 \\
Phase I DSNB & $0.02 \pm 0.00$ & $0.62 \pm 0.10$ & 0 \\
Phase II DSNB & $0.03 \pm 0.00$ & $0.91 \pm 0.15$ & 0 \\
Phase III DSNB & $0.02 \pm 0.00$ & $1.06 \pm 0.17$ & 0 \\
Total DSNB & $0.08 \pm 0.00$ & $2.58 \pm 0.26$ & 0 \\
\hline \hline
\end{tabular}

Fig. 4. We note that the intervals and best-fit value obtained with this Bayesian approach are consistent with quantities obtained by directly analyzing the likelihood space sampled by the MCMC.

In agreement with the counting analysis up to differences introduced by the statistical treatments, this result is compatible with the BSB05(GS98) model prediction and is consistent with zero hep flux. The fit yields a $68.3 \%$ HPDR credible interval for the hep flux parameter corresponding to $\Phi_{h e p}=(5.1-23) \times 10^{3} \mathrm{~cm}^{-2} \mathrm{~s}^{-1}$; as in the counting-based analysis, we define a one-sided upper limit:

$$
\Phi_{\text {hep }}<30 \times 10^{3} \mathrm{~cm}^{-2} \mathrm{~s}^{-1}(90 \% \mathrm{CI}) .
$$

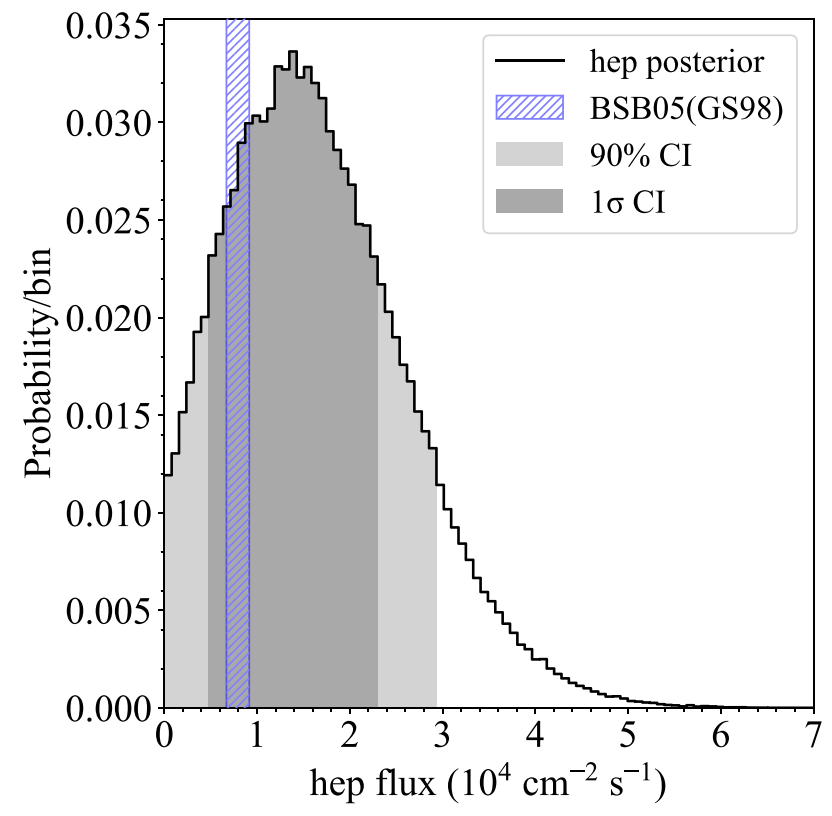

FIG. 4. The posterior distribution for the hep flux, marginalized over all other fit parameters, with the $90 \%$ and $1 \sigma$ credible intervals. The BSB05(GS98) standard solar model prediction $[7,8]$ is also shown for comparison. 

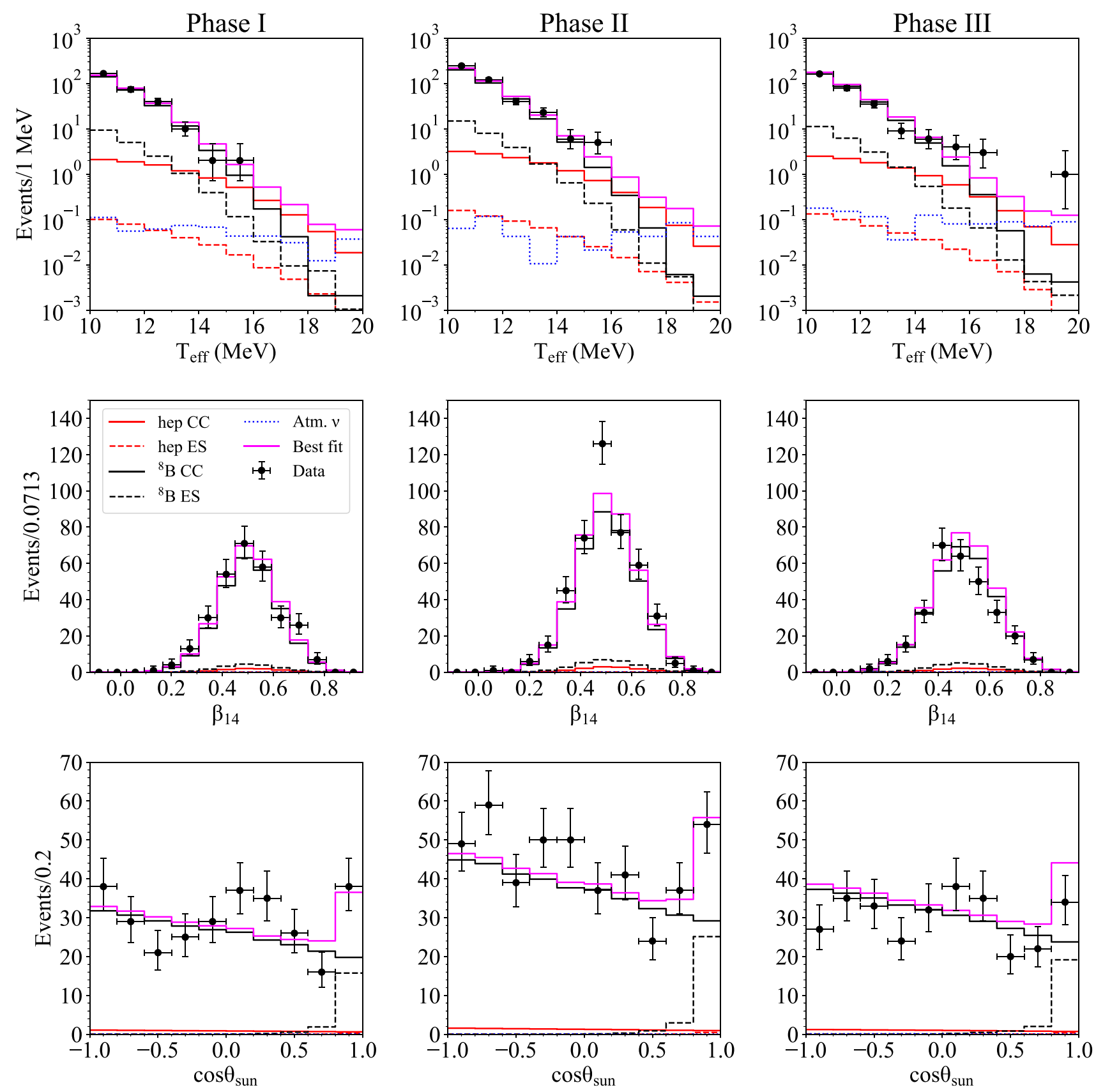

FIG. 5. Distributions of events in the full dataset compared to the best fit in the joint three-phase likelihood analysis, with projections shown for each phase and fit observable. Distributions are shown over the full energy range of the fit, 10-20 MeV. The model and systematic uncertainties are discussed in Secs. III E and III F, respectively, with the extraction of the hep flux described in Sec. IV B.

\section{CONCLUSIONS}

Data from the full SNO dataset, representing an exposure of 2.47 kilotonne years with a $\mathrm{D}_{2} \mathrm{O}$ target, has been analyzed to search for neutrinos from the hep reaction in the Sun's $p p$ chain and $\nu_{e}$ from the diffuse supernova neutrino background. In addition to increasing the exposure by a factor of 3.8 relative to the previous SNO search for these signals [16], a new spectral fit has been employed to improve the sensitivity to the hep flux.
We have performed the most sensitive search to date for the hep solar neutrino flux, the final unobserved branch of the $p p$ fusion chain. This measurement is compatible with the BSB05(GS98) model prediction of (7.93 \pm 1.23) $\times 10^{3} \mathrm{~cm}^{-2} \mathrm{~s}^{-1}$, while remaining consistent with zero hep flux, and we extract a one-sided upper limit of $\Phi_{\text {hep }}<$ $30 \times 10^{3} \mathrm{~cm}^{-2} \mathrm{~s}^{-1} 90 \% \mathrm{CI}$. In a search at energies above the solar neutrino end points, we observe no evidence for the DSNB $\nu_{e}$ flux, and set an upper limit on this flux; our 
results suggest that a $\nu_{e}$ flux larger than $\sim 30$ times the current predictions is disfavored. Upcoming experiments sensitive to DSNB $\bar{\nu}_{e}$ through inverse beta decay anticipate sensitivity at the level of model predictions [37-39]. Additionally, the DUNE experiment [40,41] and other future large detectors may offer improved sensitivity to both hep and DSNB neutrinos.

\section{ACKNOWLEDGMENTS}

This research was supported by: Canada: Natural Sciences and Engineering Research Council, Industry Canada, National Research Council, Northern Ontario Heritage Fund, Atomic Energy of Canada, Ltd., Ontario Power Generation, High Performance Computing Virtual Laboratory, Canada Foundation for Innovation, Canada Research Chairs program, Breakthrough Prize Fund at Queen's University; U.S.: Department of Energy Office of Nuclear Physics, National Energy Research Scientific Computing Center, Alfred P. Sloan Foundation, National Science Foundation, Department of Energy National Nuclear Security Administration through the Nuclear Science and Security Consortium; U.K.: Science and Technology Facilities Council (formerly Particle Physics and Astronomy Research Council); Portugal: Fundação para a Ciência e a Tecnologia. We thank the SNO technical staff for their strong contributions. We thank INCO (now Vale, Ltd.) for hosting this project in their Creighton mine. We also thank John Beacom for helpful suggestions and discussions regarding atmospheric neutrino backgrounds.

\section{APPENDIX: HIGH-ENERGY SIDEBAND}

Table VI provides details on events discussed in Sec. III F 2. These events are selected at energies $35<T_{\text {eff }}<100 \mathrm{MeV}$.

TABLE VI. Details of selected high-energy sideband events. Where associated time-correlated events are present, the time difference $\Delta t$ relative to the selected electron-like event is given, along with the reconstructed energy of the coincidence event, $E_{\text {coinc }}$.

\begin{tabular}{|c|c|c|c|c|c|c|}
\hline Phase & $N_{\text {hits }}$ & $E(\mathrm{MeV})$ & Radius (cm) & Coincidence & $\Delta t$ & $E_{\text {coinc }}$ \\
\hline I & 379 & 48.1 & 247.9 & $\gamma$ candidate & $-1.2 \mu \mathrm{s}$ & $3.9 \mathrm{MeV}$ \\
\hline I & 452 & 56.8 & 143.5 & & & \\
\hline II & 330 & 42.0 & 432.6 & & & \\
\hline II & 369 & 44.5 & 320.4 & $\mu$ candidate & $-0.8 \mu \mathrm{s}$ & $16.3 \mathrm{MeV}$ \\
\hline II & 401 & 58.3 & 104.0 & $\gamma$ candidate & $-1.7 \mu \mathrm{s}$ & $4.3 \mathrm{MeV}$ \\
\hline II & 472 & 67.5 & 173.2 & & & \\
\hline II & 400 & 57.6 & 487.7 & & & \\
\hline II & 633 & 81.9 & 405.9 & & & \\
\hline III & 313 & 43.0 & 386.6 & $\gamma$ candidate & $-1.3 \mu \mathrm{s}$ & $2.4 \mathrm{MeV}$ \\
\hline III & 348 & 47.5 & 179.4 & $\mu$ candidate & $-0.6 \mu \mathrm{s}$ & $9.4 \mathrm{MeV}$ \\
\hline III & 265 & 38.2 & 354.4 & & & \\
\hline III & 258 & 36.0 & 539.8 & $n$ candidate & $+18 \mathrm{~ms}$ & $5.5 \mathrm{MeV}$ \\
\hline III & 326 & 47.3 & 487.7 & & & \\
\hline
\end{tabular}

[1] B. T. Cleveland, T. Daily, R. J. Davis, J. R. Distel, K. Lande, C. K. Lee, P. S. Wildenhain, and J. Ullman, Astrophys. J. 496, 505 (1998).

[2] M. Altmann et al. (GNO Collaboration), Phys. Lett. B 616, 174 (2005).

[3] W. Hampel et al. (GALLEX Collaboration), Phys. Lett. B 447, 127 (1999).

[4] G. Bellini et al. (Borexino Collaboration), Phys. Rev. D 89, 112007 (2014).

[5] B. Aharmim et al. (SNO Collaboration), Phys. Rev. C 88, 025501 (2013).
[6] K. Abe et al. (Super-Kamiokande Collaboration), Phys. Rev. D 94, 052010 (2016).

[7] J. N. Bahcall, A. M. Serenelli, and S. Basu, Astrophys. J. Suppl. Ser. 165, 400 (2006).

[8] A. M. Serenelli, Nucl. Phys. B168, 115 (2007).

[9] C. Lunardini, Astropart. Phys. 79, 49 (2016).

[10] J. F. Beacom, Annu. Rev. Nucl. Part. Sci. 60, 439 (2010).

[11] W. D. Arnett, J. N. Bahcall, R.P. Kirshner, and S. E. Woosley, Annu. Rev. Astron. Astrophys. 27, 629 (1989).

[12] K. Hirata et al., Phys. Rev. Lett. 58, 1490 (1987).

[13] R. M. Bionta et al., Phys. Rev. Lett. 58, 1494 (1987). 
[14] E. Alekseev, L. Alekseeva, V. Volchenko, and I. Krivosheina, JETP Lett. 45, 461 (1987), https://ui.adsabs.harvard .edu/abs/1987PZETF..45..461A.

[15] J. F. Beacom and L. E. Strigari, Phys. Rev. C 73, 035807 (2006).

[16] B. Aharmim et al. (SNO Collaboration), Astrophys. J. 653, 1545 (2006).

[17] J. Boger et al. (SNO Collaboration), Nucl. Instrum. Methods Phys. Res., Sect. A 449, 172 (2000).

[18] Q. R. Ahmad et al. (SNO Collaboration), Phys. Rev. Lett. 89, 011301 (2002).

[19] B. Aharmim et al. (SNO Collaboration), Phys. Rev. C 72, 055502 (2005).

[20] B. Aharmim et al. (SNO Collaboration), Phys. Rev. C 81, 055504 (2010).

[21] N. J. Tagg, A. S. Hamer, B. Sur, E. D. Earle, R. L. Helmer, G. Jonkmans, B. A. Moffat, and J. J. Simpson, Nucl. Instrum. Methods Phys. Res., Sect. A 489, 178 (2002).

[22] C. Andreopoulos et al., Nucl. Instrum. Methods Phys. Res., Sect. A 614, 87 (2010).

[23] C. Andreopoulos, C. Barry, S. Dytman, H. Gallagher, T. Golan, R. Hatcher, G. Perdue, and J. Yarba, arXiv:1510 .05494 .

[24] G. D. Barr, T. K. Gaisser, P. Lipari, S. Robbins, and T. Stanev, Phys. Rev. D 70, 023006 (2004).

[25] T. K. Gaisser and T. Stanev, Phys. Rev. D 57, 1977 (1998).

[26] H. Ejiri, Phys. Rev. C 48, 1442 (1993).

[27] K. Kobayashi et al., Nucl. Phys. B139, 72 (2005).
[28] G. Battistoni, A. Ferrari, T. Montaruli, and P. R. Sala, Astropart. Phys. 23, 526 (2005).

[29] J. N. Bahcall and R. K. Ulrich, Rev. Mod. Phys. 60, 297 (1988).

[30] J. N. Bahcall, Phys. Rev. C 56, 3391 (1997).

[31] W. T. Winter, S. J. Freedman, K. E. Rehm, and J. P. Schiffer, Phys. Rev. C 73, 025503 (2006).

[32] Particle Data Group, J. Phys. G 37, 075021 (2010).

[33] A. W. P. Poon, R. J. Komar, C. E. Waltham, M. C. Browne, R. G. H. Robertson, N.P. Kherani, and H. B. Mak, Nucl. Instrum. Methods Phys. Res., Sect. A 452, 115 (2000).

[34] S. R. Seibert, A low energy measurement of the ${ }^{8} \mathrm{~B}$ solar neutrino spectrum at the Sudbury Neutrino Observatory, Ph.D. thesis, The University of Texas at Austin, Austin, TX, 2008.

[35] B. Aharmim et al. (SNO Collaboration), Phys. Rev. C 75, 045502 (2007).

[36] B. Aharmim et al. (SNO Collaboration), Phys. Rev. C 87, 015502 (2013).

[37] J. F. Beacom and M. R. Vagins, Phys. Rev. Lett. 93, 171101 (2004).

[38] L. Marti-Magro (Super-Kamiokande Collaboration), Proc. Sci., ICHEP2018 (2019) 009.

[39] F. An et al., J. Phys. G 43, 030401 (2016).

[40] F. Capozzi, S. W. Li, G. Zhu, and J. F. Beacom, Phys. Rev. Lett. 123, 131803 (2019).

[41] DUNE Collaboration, arXiv:2002.03005. 